\title{
MULTIPLICITY RESULTS FOR SEMILINEAR ELLIPTIC BOUNDARY VALUE PROBLEMS IN BESOV AND TRIEBEL-LIZORKIN SPACES
}

\author{
by L. PÄIVÄRINTA and T. RUNST
}

(Received 29th October 1989)

\begin{abstract}
The paper deals with superlinear elliptic boundary value problems depending on a parameter. Given appropriate hypotheses concerning the asymptotic behaviour of the nonlinearity, we prove lower bounds on the number of solutions. The results generalize a theorem due to Lazer and McKenna within the framework of quasi-Banach spaces of Besov and Triebel-Lizorkin spaces.
\end{abstract}

1980 Mathematics subject classification (1985 Revision): 46E, 35J, 35B, 58E.

\section{Introduction}

Let $\Omega$ be a smooth bounded domain in $\mathbb{R}^{n}$ (the Euclidean $n$-space) with boundary $\partial \Omega$. Then we consider semilinear elliptic boundary value problems of the type

$$
\begin{array}{ll}
L u=f(u)+h(x) & \text { in } \Omega \\
u=0 & \text { on } \partial \Omega,
\end{array}
$$

where $L$ is a second-order uniformly elliptic, formally self-adjoint linear operator and $h$ belongs to a function space of Triebel-Lizorkin and Besov type, respectively. Let $0<\lambda_{1}<\lambda_{2} \leqq \cdots \leqq \lambda_{k} \leqq \cdots$ denote the eigenvalues of $L$ with Dirichlet boundary value conditions. Here $f$ is a sufficiently smooth real-valued function with linear growth at infinity, and more precisely:

$$
a \leqq f^{\prime}(t) \leqq b \text { for all } t \in[-\infty, \infty], \text { where } f^{\prime}( \pm \infty)=\lim _{t \rightarrow \pm \infty} f^{\prime}(t)
$$

It is known that our problem (1.1) admits multiple solutions depending on the interaction between the values of $f^{\prime}$ and the spectrum of $L$ if $h$ belongs to the Hölder spaces $C^{\alpha}(\bar{\Omega}), 0<\alpha<1$. First, note that if $[a, b]$ contains no eigenvalue $\lambda_{k}$, then $(1.1)$ is uniquely solvable in $C^{2+\alpha}(\bar{\Omega})$, see Dolph [6].

The first result, where the nonlinearity $f$ meets the first eigenvalue, was proved by Ambrosetti and Prodi [2]. They considered the case in which the range of $f^{\prime}$ contains only the first (simple) eigenvalue $\lambda_{1}$. They showed that the conditions $0<f^{\prime}(-\infty)<\lambda_{1}$, $\lambda_{1}<f^{\prime}(\infty)<\lambda_{2}$ and $f^{\prime \prime}>0$ on $(-\infty, \infty)$ imply the existence of a closed connected 
$C^{1}$-manifold $M_{1}$ of codimension 1 in the Banach space $C^{\alpha}(\bar{\Omega})$ such that $C^{\alpha}(\bar{\Omega}) \backslash M_{1}$ has exactly two components $M_{0}$ and $M_{2}$ with the property that

$$
\begin{aligned}
-\Delta u & =f(u)+h(x) & & \text { in } \Omega \\
u & =0 & & \text { on } \partial \Omega
\end{aligned}
$$

has no solution if $h \in M_{0}$, exactly one solution if $h \in M_{1}$, and exactly two solutions if $h \in M_{2}$. A corresponding result within the framework of Besov and Triebel-Lizorkin spaces was proved in Geisler and Runst [12]. Manes and Micheletti [19] replaced the condition $0<f^{\prime}(-\infty)<\lambda_{1}$ by $-\infty<f^{\prime}(-\infty)<\lambda_{1}$.

The next important result was obtained by Kazdan and Warner [16] for more general functions $f$. For example, they showed if one decomposes $h=h_{1}+t \varphi_{1}\left(\varphi_{1}\right.$ : normalized eigenfunction to $\lambda_{1}, h_{1}$ : smooth function which satisfies $\left.\int_{\Omega} h_{1}(x) \varphi_{1}(x) d x=0\right)$, then there exists $t_{0}=t_{0}\left(h_{1}\right)$ such that (1.1) has no solution for $t>t_{0}$ and at least one solution for $t<t_{0}$. Simultaneously, Dancer [4] and Amann and Hess [1] showed that if $f$ satisfies $f^{\prime}(-\infty)<\lambda_{1}<f^{\prime}(\infty)$ and $f^{\prime}$ is bounded on $[0, \infty)$, then (1.1) has at least two solutions if $h=h_{1}+t \varphi_{1}$ and $t<t_{0}\left(h_{1}\right)$ and at least one solution if $t=t_{0}$, see also Berger and Podolak [3]. In [17], Lazer and McKenna showed that if $f^{\prime}(-\infty)<\lambda_{1}$ and $\lambda_{2 k}<f^{\prime}(\infty)<\lambda_{2 k+1}(k \geqq 1)$, then there exists $t_{1} \leqq t_{0}$ such that (1.1) has at least three solutions if $h=h_{1}+t \varphi_{1}$ and $t<t_{1}$ (perform the change of variable $u \rightarrow-u$ in order to bring the problem considered in [17] to the present setting). Further results in this direction can be found in Hess and Ruf [13], Ruf [21] and Solimini [23]. Furthermore, Lazer and McKenna obtained in [18] that if $f^{\prime}(-\infty)<\lambda_{1}$ and $\lambda_{2}<f^{\prime}(\infty) \leqq \lambda_{3}$, then (1.1) has at least four solutions if $h=h_{1}+t \varphi_{1}$ and $t$ is sufficiently small. There was also shown that if $\lambda_{3}$ has odd multiplicity, there exists $\beta>\lambda_{3}$ such that if $f^{\prime}(-\infty)<\lambda_{1}, \lambda_{3}<f^{\prime}(\infty) \leqq$ $\beta$ and $h=h+t \varphi_{1}$, then (1.1) has at least five solutions for $t$ sufficiently small.

In this paper, we consider equations of type (1.1) within the framework of TriebelLizorkin spaces, $F_{p, q}^{s}$, and Besov spaces, $B_{p, q}^{s}$, with methods going back to [17]. For $0<q<1$ and/or $0<p<1, B_{p, q}^{s}$ and $F_{p, q}^{s}$ become quasi-Banach spaces (see 2.4). For instance, quasi-Banach spaces are not locally convex, in general. Hence Schauder's fixed point theorem is not applicable in these cases. Lazer and McKenna obtained their results in [17] using the Leray-Schauder degree. Klee [14] proved that it is possible to develop the Leray-Schauder theory in so-called admissible topological spaces. Up to now, it is an open problem whether every quasi-Banach space is admissible in the sense of Klee. However, in Franke and Runst [9], we obtained that the function spaces of Triebel-Lizorkin and Besov type are admissible. Hence we can carry over the theory of [17].

The paper is organized as follows. In Section 2, we describe the preliminaries (function spaces on smooth domains, mapping properties of linear differential operators and of nonlinear operators generated by smooth functions, results of the LeraySchauder theory). Section 3 deals with the number of solutions of (1.1). The first result is an improvement of one obtained in Drabek and Runst [7]; the second is a generaliza- 
tion of the main result for Hölder spaces in Lazer and McKenna [17] formulated now within the framework of Besov and Triebel-Lizorkin spaces.

\section{Preliminaries}

\subsection{Spaces}

Let $\mathbb{R}^{n}$ be the real Euclidean $n$-space. In the following, we list some properties of the spaces $B_{p, q}^{s}$ and $F_{p, q}^{s}$, see Triebel [24] for details.

Let $S$ be the Schwartz space of all complex-valued rapidly decreasing infinitely differentiable functions on $\mathbb{R}^{n}$ and let $S^{\prime}$ be its topological dual. The Fourier transform and its inverse on $S^{\prime}$ is denoted by $F$ and $F^{-1}$, respectively. Now let $\varphi \in S$ be a real-valued even function with respect to the origin such that $\varphi(x)=\varphi(-x)$ if $x \in \operatorname{supp} \varphi \subset\left\{y \in \mathbb{R}^{n},|y| \leqq 2\right\}$ and $\varphi(x)=1$ if $|x| \leqq 1$. Then we define a sequence $\left\{\varphi_{j}\right\}_{j=0}^{\infty}$ of functions by

$$
\varphi_{0}(x)=\varphi(x), \quad \varphi_{j}(x)=\varphi\left(2^{-j} x\right)-\varphi\left(2^{-j+1} x\right), \quad j=1,2, \ldots
$$

for each $x \in \mathbb{R}^{n}$. We have $\sum_{j=0}^{\infty} \varphi_{j}(x)=1$ for all $x \in \mathbb{R}^{n}$.

If $-\infty<s<\infty, 0<p, q \leqq \infty$, then by definition

$$
B_{p, q}^{s}\left(\mathbb{R}^{n}\right)=\left\{f \in S^{\prime},\left\|f \mid B_{p, q}^{s}\right\|=\left(\sum_{j=0}^{\infty} 2^{j s q}\left\|F^{-1} \varphi_{j} F f \mid L_{p}\left(\mathbb{R}^{n}\right)\right\|^{q}\right)^{1 / q}<\infty\right\}
$$

and if $p<\infty$,

$$
F_{p, q}^{s}\left(\mathbb{R}^{n}\right)\left\{f \in S^{\prime},\left\|f\left|F_{p, q}^{s}\|=\|\left(\sum_{j=0}^{\infty} 2^{j s q}\left|F^{-1} \varphi_{j} F f(\cdot)\right|^{q}\right)^{1 / q}\right| L_{p}\left(\mathbb{R}^{n}\right)\right\|<\infty\right\}
$$

(usual modification if $p=\infty$ and/or $q=\infty$ ).

It can be shown (see Triebel [24]) that $B_{p, q}^{s}\left(\mathbb{R}^{n}\right)$ and $F_{p, q}^{s}\left(\mathbb{R}^{n}\right)$ are quasi-Banach spaces (Banach spaces if $\min (p, q) \geqq 1$ ).

Remark 2.1. By means of the fact that $\varphi$ is a real-valued even function we can introduce the real part of the spaces $B_{p, q}^{s}\left(\mathbb{R}^{n}\right)$, etc., denoted by $\tilde{B}_{p, q}^{s}\left(\mathbb{P}^{n}\right), \ldots$ (for exact definitions see Franke and Runst [9, Subsection 3.2]).

Remark 2.2. These two scales of function spaces include many well-known classical spaces. We give some examples, for details see Triebel [24].

Let $s>0$, then $B_{\infty, \infty}^{s}\left(\mathbb{R}^{n}\right)=\mathscr{C}^{s}\left(\mathbb{R}^{n}\right)$ (Zygmund space). If $s>0$ is not an integer, then $B_{\infty, \infty}^{s}\left(\mathbb{R}^{n}\right)=C^{s}\left(\mathbb{R}^{n}\right)$ (Hölder space). Let $1<p<\infty,-\infty<s<\infty$, then $F_{p, 2}^{s}\left(\mathbb{R}^{n}\right)=H_{p}^{s}\left(\mathbb{R}^{n}\right)$ (Bessel-potential space). If $s$ is a natural number, then $F_{p, 2}^{s}\left(\mathbb{R}^{n}\right)=W_{p}^{s}\left(\mathbb{R}^{n}\right)$ (Sobolev space). 
For dealing with boundary value problems it is useful to define Besov and TriebelLizorkin spaces on domains. Let $\Omega$ be a bounded $C^{\infty}$-domain in $\mathbb{R}^{n}$ with boundary $\partial \Omega$. Then one can introduce the spaces $B_{p, q}^{s}(\partial \Omega)$ and $F_{p, q}^{s}(\partial \Omega)$ by standard procedures via local charts (see Triebel [24, Subsection 3.2.2]). The spaces $B_{p, q}^{s}(\Omega)$ and $F_{p, q}^{s}(\Omega)$ are defined usually by the restriction method (see Triebel [24, Subsection 3.3.1] and [25]).

(i) Let $0<p_{0}, p_{1}, q_{0}, q_{1} \leqq \infty$ and $-\infty<s_{1}<s_{0}<\infty$. Then

$$
D(\Omega) \subset B_{p 0, q 0}^{s o}(\Omega) \subset B_{p_{1}, q_{1}}^{s_{1}}(\Omega) \subset D^{\prime}(\Omega)
$$

$$
\text { if } s_{0}-\left(n / p_{0}\right)>s_{1}-\left(n / p_{1}\right) \text {. }
$$

(ii) Let $0<p_{0}, p_{1}<\infty, 0<q_{0}, q_{1} \leqq \infty$ and $-\infty<s_{1}<s_{0}<\infty$. Then

$$
D(\Omega) \subset F_{p 0, q_{0}}^{s_{0}}(\Omega) \subset F_{p_{1}, q_{1}}^{s_{1}}(\Omega) \subset D^{\prime}(\Omega)
$$

$$
\text { if } s-\left(n / p_{0}\right)>s_{1}-\left(n / p_{1}\right) \text {. }
$$

Here $D(\Omega)$ denotes, as usual, the collection of all complex-valued infinitely differentiable functions $f$ in $\mathbb{R}^{n}$ with supp $f \subset \Omega$, and $D^{\prime}(\Omega)$ is the dual space.

\subsection{Traces and linear elliptic differential operators}

Let $\Omega$ be a bounded $C^{\infty}$-domain in $\mathbb{R}^{n}$ and let $f$ be a function defined in $\Omega$ belonging to some function spaces of the above type. In the following, $R$ denotes the restriction operator given by $R f=f \mid \partial \Omega$. The following results are known (see Triebel [24, Subsection 3.3.3] and Franke [8]).

If $0<p, q \leqq \infty$ and $s>s^{*}:=(n-1)(1 / \min (p, 1)-1)+1 / p$, then $R$ is a linear and continuous mapping from $B_{p, q}^{s}(\Omega)$ onto $B_{p, q}^{s-1 / p}(\partial \Omega)$ and if $p>\infty$, then $R$ is a linear and continuous mapping from $F_{p, q}^{s, q}(\Omega)$ onto $B_{p, q}^{s-1 / p}(\partial \Omega)$.

Let

$$
L=-\sum_{i, j=1}^{n} \frac{\partial}{\partial x_{i}} a_{i j}(x) \frac{\partial}{\partial x_{j}}, a_{i j}(x) \in \widetilde{C}^{\infty}(\bar{\Omega})
$$

satisfying $a_{i j}(x)=a_{i j}(x)$, be a second-order uniformly elliptic operator. In this paper, we only consider the corresponding homogeneous Dirichlet problem. We introduce (for admissible couples $(s, p)$ )

$$
B_{p, q, 0}^{s}(\Omega)=\left\{f \in B_{p, q}^{s}(\Omega), f \mid \partial \Omega=0\right\}
$$

and

$$
F_{p, q, 0}^{s}(\Omega)=\left\{f \in F_{p, q}^{s}(\Omega), f \mid \partial \Omega=0\right\}
$$

Then the following result can be found in Franke [8] (see also Triebel [24, Subsection 
3.3.3]), for $0<q \leqq \infty$ and $s>s^{*}$. If $0<p \leqq \infty$ then $L$ yields an isomorphic mapping from $B_{p, q, 0}^{s}(\Omega)$ onto $B_{p, q}^{s-2}(\Omega)$. If $0<p<\infty$ then $L$ yields an isomorphic mapping from $F_{p, q, 0}^{s}(\Omega)$ onto $F_{p, q}^{s-2}(\Omega)$.

By Fucik [11, Theorem 34.10], we obtain the following result. If $\lambda_{1}$ denotes the smallest eigenvalue of $\left.L\right|_{\dot{\beta}_{2,2}(\Omega)}$ with Dirichlet condition, then it holds $\lambda_{1}>0$ and $\lambda_{1}$ is a simple eigenvalue $\left(\dot{B}_{p, q}^{s}(\Omega)\right.$ denotes the completion of $D(\Omega)$ in $\left.B_{p, q}^{s}(\Omega)\right)$. Furthermore, there exists a unique normed positive eigenfunction $\varphi_{1} \in \widetilde{C}^{\infty}(\bar{\Omega})$ to $\lambda_{1}$ with $\varphi_{1}(x)>0$ in $\Omega, L \varphi_{1}=\lambda_{1} \varphi_{1},\left(\partial \varphi_{1} / \partial v\right)<c<0$ on $\partial \Omega$ where $v$ is the normal, and $\int_{\Omega} \varphi_{1}(x)^{2} d x=1$.

In order to prove our main result we need some facts about sub- and supersolutions.

Definition 2.1. A distribution $\psi \in \tilde{D}^{\prime}(\Omega)$ is said to be non-negative if $\psi(\varphi) \geqq 0$ for any $\varphi \in \tilde{D}(\Omega)$ with $\varphi \geqq 0$.

Remark 2.3. The set of non-negative distributions is $\sigma\left(D^{\prime}(\Omega), D(\Omega)\right)$-closed.

Definition 2.2. A function $u \in \widetilde{C}(\bar{\Omega})$ is said to be a supersolution (subsolution) of (1.1) if $L u \geqq f(u)+h(x)$ in $\Omega(L u \leqq f(u)+h(x)$ in $\Omega)$ in the above sense and $u \mid \partial \Omega=0$.

In Section 3 we use the following maximum principle.

Lemma 2.1. Let $v \in \bigcup_{e>0} B_{\infty, \infty}^{e}(\Omega)$ and let $\mu>-\lambda_{1}$. If $v \mid \partial \Omega=0$ and $(L+\mu) v \geqq 0$ (in the above sense of distributions) then $v \geqq 0$ holds.

Proof. Step 1. Let $w \in \widetilde{B}_{\infty, \infty}^{e-2}(\Omega), 0<\varepsilon<1$, be non-negative. If $\psi \in \tilde{C}^{\infty}(\bar{\Omega}), \psi \mid \partial \Omega=0$ then $\psi \in \widetilde{B}_{\infty, \infty}^{2-\varepsilon}(\Omega)$. In Franke and Runst [10] (see also Triebel [24, Subsection 3.4.3]), it is proved: If $\psi$ is non-negative, then $\psi$ can be approximated in $\widetilde{B}_{1,1}^{2-\varepsilon}(\Omega)$ by nonnegative $\tilde{C}_{0}^{\infty}(\Omega)$-functions. Hence $\psi(w)$ is well defined (for the dual space of $\left(\tilde{B}_{1,1}^{2-e}():\left(\tilde{\bar{B}}_{1,1}^{2-e}(\Omega)\right)^{\prime}=\tilde{B}_{\infty, \infty}^{e-2}(\Omega)\right)$ and non-negative. If $f \in \tilde{C}_{0}^{\infty}(\Omega)$ is non-negative, then there exists a non-negative $g \in \widetilde{C}^{\infty}(\bar{\Omega})$ with $g \mid \partial \Omega=0,(L+\mu) g=f$ (see Fucik [11, Chapter 34]). According to $\int_{\Omega} \psi(x) f(x) d x=\int_{\Omega} \varphi(x) g(x) d x$ we obtain the following: If $\varphi \in \widetilde{C}_{0}^{\infty}(\Omega)$, $\psi \mid \partial \Omega=0, \psi$ is non-negative if $\varphi$ is. Here we used the fact that $L$ is formally self-adjoint, i.e.

$$
\int_{\Omega} L u_{1}(x) u_{2}(x) d x=\int_{\Omega} u_{1}(x) L u_{2}(x) d x
$$

if $u_{1}, u_{2} \in \bar{C}^{\infty}(\bar{\Omega}), u_{1}\left|\partial \Omega=u_{2}\right| \partial \Omega=0$.

Step 2. Let $v$ be the same as in the formulation of Lemma 2.1. Let $\varphi \in \bar{C}_{0}^{\infty}(\Omega)$ be non-negative, $\varphi=(L+\mu) \psi$ with $\psi \in \tilde{C}^{\infty}(\Omega)$, non-negative and $\psi \mid \partial \Omega=0$. Then an easy limiting argument proves

$$
\int_{\Omega} \varphi(x) v(x) d x=\int_{\Omega}(L+\mu) \psi(x) v(x) d x
$$




$$
=\int_{\Omega} \psi(x)(L+\mu) v(x) d x \geqq 0
$$

which completes our proof.

Remark 2.4. The following version of the strong maximum principle holds also: Suppose that $v \in \bigcup_{e>0} \tilde{B}_{\infty, \infty}^{e}(\Omega), c, \mu \in \mathbb{R}$ with $\mu>-\lambda_{1}$. If $v \mid \partial \Omega=0$ and $(L+\mu) v \geqq c>0$ (in the above sense of distributions) then $v>0$ in $\Omega$ holds.

\subsection{Mapping properties}

In this subsection, we list some results which can be found in Runst [22, Subsection 5.4]. $C^{\rho}$ denotes as usual the classical Hölder space if $\rho>0$ is not an integer and the well-known Banach space of differentiable functions if $\rho>0$ is an integer. As mentioned above, $B_{\infty, \infty}^{\rho}=\mathscr{C}^{\rho}$ if $\rho>0$.

Lemma 2.2. Let $f \in \tilde{C}^{\rho+1}, \rho>\max (1, s)$. Then $u \rightarrow f(u)$ is a completely continuous mapping

$$
\begin{array}{ccc}
\text { from } \tilde{F}_{p, q}^{s+\varepsilon}(\Omega) \cap L_{\infty}(\Omega) & \text { into } & \tilde{F}_{p, q}^{s}(\Omega) \cap L_{\infty}(\Omega) \\
\left(\text { from } \tilde{B}_{p, q}^{s+\varepsilon}(\Omega) \cap L_{\infty}(\Omega)\right. & \text { into } & \left.\widetilde{B}_{p, q}^{s}(\Omega) \cap L_{\infty}(\Omega)\right)
\end{array}
$$

if $0<p<\infty(0<p \leqq \infty), 0<q \leqq \infty, s>n((1 / \min (p, 1))-1)$ and $\varepsilon>0$.

Furthermore, there exists a function $g_{f}, g_{f}:[0, \infty) \rightarrow[0, \infty)$, which is independent of $u$ such that

$$
\begin{gathered}
\left\|f(u)\left|F_{p, q}^{s}(\Omega)\left\|\leqq g_{f}\left(\left\|u \mid L_{\infty}\right\|\right)\right\| u\right| F_{p, q}^{s}\right\| \\
\left(\left\|f(u)\left|B_{p, q}^{s}(\Omega)\left\|\leqq g_{f}\left(\left\|u \mid L_{\infty}\right\|\right)\right\| u\right| B_{p, q}^{s}\right\|\right) .
\end{gathered}
$$

Remark 2.5. This result is a consequence of Runst [22, Subsection 5.4] and (2.1/2).

\subsection{The Leray-Schauder degree}

Let $A$ be a (real or complex) linear vector space. $\|\cdot \mid A\|$ is said to be a quasi-norm if $\|\cdot \mid A\|$ satisfies the usual conditions of a norm with the exception of the triangle inequality, which is replaced by

$$
\left\|a_{1}+a_{2} \mid A\right\| \leqq c\left(\left\|a_{1}\left|A\|+\| a_{2}\right| A\right\|\right)
$$

i.e. there exists a positive number $c$ such that (2.3) holds for all $a_{1} \in A$ and all $a_{2} \in A$. Of course $c \geqq 1$. (If $c=1$ is admissible, then $A$ is a normed space.) A quasi-normed space is 
said to be a quasi-Banach space if it is complete. By a theorem due to Rolewicz (see Köthe [15, Subsection 18.10]) we may assume without loss of generality that

$$
\left\|a_{1}+a_{2}\left|A\left\|^{\lambda} \leqq\right\| a_{1}\right| A\right\|^{\lambda}+\left\|a_{2} \mid A\right\|^{\lambda}
$$

holds for suitable $\lambda, 0<\lambda \leqq 1$. This makes $A$ into a linear metric space with translation invariant metric

$$
d_{A}\left(a_{1}, a_{2}\right):=\left\|a_{1}-a_{2} \mid A\right\|^{\lambda}
$$

Definition 2.3. Suppose that $A$ is a quasi-normed space of type $\lambda$. Such a space is said to be admissible if for every compact subset $K \subset A$ and for every $\varepsilon>0$ there exists a continuous mapping $T: K \rightarrow A$ such that $T(K)$ is contained in a finite-dimensional subset of $A$ and $x \in K$ implies $\|T x-x \mid A\| \leqq \varepsilon$.

Remark 2.6. We introduced the notation "admissible" in the sense of Klee [14], see also Riedrich [20, Subsection 4.1].

In the following, we use essentially the fact that the spaces considered here are admissible. The next lemma has turned out to be very helpful.

Lemma 2.3 (Franke and Runst [9, Subsection 3.1]). Let $A$ and $B$ be (real or complex) quasi-normed spaces. Furthermore, let $T_{0}: A \rightarrow B$ and $T_{1}: B \rightarrow A$ be continuous mappings. Suppose that $T_{1}$ is uniformly continuous on every bounded set and let $T_{1} T_{0}=I_{A}$ (identity of $A$ ). Then if $B$ is admissible then $A$ is also admissible.

By Riedrich [20, Subsection 4.2] every normed space is admissible. Furthermore, the quasi-Banach spaces $B_{p, q}^{s}$ and $F_{p, q}^{s}$ are of type $\lambda=\min (p, q, 1)$. (If $\lambda=1$, then they become Banach spaces.) Applying Lemma 2.3 one can prove the following result.

Lemma 2.4 (Franke and Runst [9, Subsection 3.2]). Let $0<p, q \leqq \infty$ and $-\infty<s<\infty$.

(i) The spaces $B_{p, q}^{s}\left(\mathbb{R}^{n}\right), \quad \tilde{B}_{p, q}^{s}\left(\mathbb{R}^{n}\right), \quad B_{p, q}^{s}(\Omega), \quad \widetilde{B}_{p, q}^{s}(\Omega), \quad B_{p, q}^{s}(\partial \Omega)$ and $\tilde{B}_{p, q}^{s}(\partial \Omega)$ are admissible.

(ii) Let $p<\infty$, then the spaces $F_{p, q}^{s}\left(\mathbb{R}^{n}\right), \tilde{F}_{p, q}^{s}\left(\mathbb{R}^{n}\right), F_{p, q}^{s}(\Omega), \tilde{F}_{p, q}^{s}(\Omega), F_{p, q}^{s}(\partial \Omega)$ and $\tilde{F}_{p, q}^{s}(\partial \Omega)$ are admissible.

Suppose that $X$ is an admissible quasi-Banach space, $B$ is an open and bounded subset of $X, f: \bar{B} \rightarrow X$ is a completely continuous mapping and $y \notin(I-f)(\partial B)$. On these admissible triplets $(I-f, B, y)$ one can now introduce the Leray-Schauder degree denoted by $d_{\mathrm{LS}}(I-f, B, y)$. Then the following properties hold (also in admissible topological spaces). 
Lemma 2.5 (Riedrich [20, Subsection 4.3]). Let $X$ be an admissible quasi-Banach space. Then we have

(a) $d_{\mathrm{Ls}}(I, B, y)=\left\{\begin{array}{l}0 \text { if } y \in X \backslash \bar{B} \\ 1 \text { if } y \in B\end{array}\right.$.

(b) If $d_{\mathrm{Ls}}(I-f, B, y) \neq 0$, then there exists a solution $x \in B$ such that $(I-f)(x)=y$ holds.

(c) $d_{\mathrm{LS}}(I-f, B, y)=d_{\mathrm{LS}}\left(I-f, B_{1}, y\right)+d_{\mathrm{LS}}\left(I-f, B_{2}, y\right)$ whenever $B_{1}$ and $B_{2}$ are disjoint open subsets of $B$ such that $y \notin(I-f)\left(\bar{B} \backslash\left(B_{1} \cup B_{2}\right)\right)$.

(d) $d_{\mathrm{LS}}(I-H(t, \cdot), B, y)$ is independent of $t \in[0,1]$ whenever $H:[0,1] \times \bar{B} \rightarrow X$ is a completely continuous mapping and $y \notin(I-H(t, \cdot))(\partial B)$ on $[0,1]$ (invariance under homotopy).

In what follows, let $X$ be an admissible quasi-Banach space. If $x_{0}$ is an isolated fixed point of $f$ (i.e. $(I-f)\left(x_{0}\right)=0$ and $0 \neq f(x)$ in $B_{r}\left(x_{0} \backslash\left\{x_{0}\right\}\right.$, where $B_{r}\left(x_{0}\right)=\left\{y \in X,\left\|y-x_{0} \mid X\right\|<r\right\}$ and $r$ is small enough), then we know that $d_{\mathrm{LS}}\left(I-f, B_{\rho}\left(x_{0}\right), 0\right)$ is constant for all $\rho \in(0, r)$. This number is called the index of $x_{0}$ and is denoted by $i\left(I-f, x_{0}\right)$. Furthermore, we need some results about the Leray-Schauder degree of completely continuous linear operators acting in admissible quasi-Banach spaces.

Theorem 2.1. Let $X$ be a real admissible quasi-Banach space, $L$ be a completely continuous linear operator acting in $X, 0 \neq \lambda \in R$ and $\lambda^{-1}$ is not an eigenvalue of $L$. Then $d_{\mathrm{LS}}\left(I-\lambda L, B_{R}(0), 0\right)=(-1)^{a(\lambda)}$, where $a(\lambda)$ is the sum of the algebraic multiplicities of the eigenvalues $\mu$ satisfying $\mu \lambda>1$, and $a(\lambda)=0$ if $L$ has no eigenvalues of this $k$ ind.

Proof. Step 1. By Williamson [26, p. 155] we know that there exists a smallest natural number $k=k(\lambda)$ such that $\operatorname{ker}\left[(I-\lambda L)^{k}\right]=\operatorname{ker}\left[(I-\lambda L)^{k+1}\right], \operatorname{dim} \operatorname{ker}\left[(I-\lambda L)^{k}\right]<$ $\infty, R\left[(I-\lambda L)^{k}\right]$ is closed and $X=\operatorname{ker}\left[(I-\lambda L)^{k}\right]+R\left[(I-\lambda L)^{k}\right]=: N_{k}^{\lambda}+R_{k}^{\lambda}$. Furthermore, we have $N_{k}^{\lambda} \cap R_{k}^{\lambda}=\{0\}$. Hence we obtain $X=N_{k}^{\lambda} \oplus R_{k}^{\lambda}$. Since $(I-\lambda L)^{k} L=(I-\lambda L)^{k}$ we see that $N_{k}^{\lambda}$ and $R_{k}^{\lambda}$ are invariant under $L,\left.L\right|_{R_{k}^{\lambda}}$ is one-to-one and $L R_{k}^{\lambda}=R_{k}^{\lambda}$. Hence $\left.L\right|_{R_{k}^{\lambda}}$ is a homeomorphism onto $R_{k}$. Furthermore, every eigenvalue $\lambda_{0} \neq 0$ is an isolated one. To prove it let $\tilde{L}_{\lambda}=L-\left.\lambda I\right|_{R_{k} 0}, \mu_{0}=\left(1 / \lambda_{0}\right)$. By the properties of $L$ there exists a $c>0$ such that $\left\|L_{i_{0}}|x| X\right\| \geqq c\|x \mid X\|$. Hence we get $\left\|\tilde{L}_{\lambda} x\left|X\left\|\geqq\left(\tilde{c}-\left|\lambda-\lambda_{0}\right|\right)\right\| x\right| X\right\|$, i.e. $\tilde{L}_{\dot{\lambda}}$ is one-toone for $\left|\lambda-\lambda_{0}\right|<\tilde{c}$. On the other hand, $\lambda_{0}$ is the only eigenvalue of $L$ on $N_{k}^{\mu_{0}}$. Indeed, $L_{\lambda} x=0$ for some $x \in N_{k}^{\mu_{0}}$ implies $(L-\lambda I) x=\left(\lambda-\lambda_{0}\right) x$ and therefore $0=-\lambda_{0}^{k}\left(I-\mu_{0} L\right)^{k} x=$ $\left(\lambda-\lambda_{0}\right)^{k}$ for $k=k(\lambda)$, i.e. $x=0$ if $\lambda \neq \lambda_{0}$.

Step 2. Now we can prove the above result. We may assume $\lambda=1$ for simplicity. By Step 1 there are at most finitely many eigenvalues $\mu_{1}, \ldots, \mu_{m}$ oi $L$ such that $\mu_{i} \geqq 1$. Let $Y=N\left(\mu_{1}\right) \oplus \cdots \oplus N\left(\mu_{m}\right)$ and $Z=\bigcap_{i=1}^{m} R\left(\mu_{i}\right)$, where $N\left(\mu_{i}\right)=N_{k_{i}\left(\mu_{i}\right)}^{\mu_{i}}$ and $R\left(\mu_{i}\right)=R_{k_{i}\left(\mu_{i}\right)}^{\mu_{i}}$. It is straightforward to see that $X=Y \oplus Z$ holds. Now, any $x \in X$ can be written as $x=y+z$ with $y \in Y$ and $z \in Z$. It holds that $H(t, x):=(1-t) L(y+z)+2 t y \neq x$ for all $x \in \partial B_{r}(0)$ and 
$0 \leqq t \leqq 1$ because $H(t, x)=x$ implies $(1-t) L y+2 t y=y$ and $(1-t) L z=z$. Hence we would obtain $0<t<1, L y=((1-2 t) /(1-t)) y$ and $L z=(1 /(1-t)) z$. Notice that $(1-2 t) /(1-t)<1$ for all $t \in(0,1)$. By the properties of $Y$ and $Z$ we get $y=z=0$, i.e. a contradiction to $x \in \partial B_{r}(0)$. Applying Lemma 2.5 it follows that

$$
\begin{aligned}
d_{\mathrm{LS}}\left(I-L, B_{r}(0), 0\right) & =d_{\mathrm{LS}}\left(I-H(0, \cdot), B_{r}(0), 0\right) \\
& =d_{\mathrm{LS}}\left(I-H(1, \cdot), B_{r}(0), 0\right)=d_{\mathrm{LS}}\left(-y, B_{r}(0), 0\right) \\
& =\operatorname{sgn} \operatorname{det}\left(-\left.I\right|_{Y}\right)=(-1)^{a(1)},
\end{aligned}
$$

where $a(1)=\operatorname{dim} Y$. Here we used the properties of the Leray-Schauder degree in finite dimensions (see Zeidler [27, Subsection 12.5]). Finally, if $a(1)=0$, we may consider the homotopy $H(t, x)$ : $=(1-t) L x$.

Remark 2.7. Theorem 2.1 is a generalization of the so-called index theorem to admissible quasi-Banach spaces. We used an idea similar to one due to Zeidler [27, Subsection 14.2], see also Deimling [5, Subsection 8.6].

Corollary 2.1. Let $X$ be a real admissible quasi-Banach space, let $f: B_{r}\left(x_{0}\right) \subset X \rightarrow X$ be a completely continuous mapping with $f\left(x_{0}\right)=x_{0}$. Furthermore, $f$ is (Frechet-) differentiable at $x_{0}$ and $\lambda=1$ is not an eigenvalue of $L:=f^{\prime}\left(x_{0}\right)$. Then $x_{0}$ is an isolated fixed point of $f$ and $i\left(I-f, x_{0}\right)=d_{\mathrm{LS}}\left(I-L, B_{r}(0), 0\right)$.

Proof. The proof is essentially the same as for Banach spaces (see Zeidler [27, Korollar 14.1]).

\section{Boundary value problems}

\subsection{On the existence of solutions}

Let $\Omega$ be a bounded $C^{\infty}$-domain in $\mathbb{R}^{n}$ and let

$$
\begin{array}{ll}
L u=f(u)+h_{1}(x)+t \varphi_{1}(x) & \text { in } \Omega \\
u=0 & \text { on } \partial \Omega
\end{array}
$$

be a semilinear elliptic boundary value problem, where $L$ and $\varphi_{1}$ are the same as in 2.2 . The function $h_{1}$ belongs to a real Besov and Triebel-Lizorkin space, respectively, and satisfies $\int_{\Omega} h_{1}(x) \varphi_{1}(x) d x=0$.

Theorem 3.1. Let $0<p, q \leqq \infty, s>(n / p), t \in \mathbb{R}$ and $f \in \bar{C}^{\rho+1}(\mathbb{R}), \rho>\max (1, s)$, satisfying the conditions:

(f1) there exists a constant $c$ such that $f(x)-\lambda_{1} x>c$, 
(f2) $\lim _{r \rightarrow-\infty} \frac{f(r)}{r}<\lambda_{1}$.

(i) Let $h_{1} \in \tilde{B}_{p, q}^{s-2}(\Omega) \cap L_{\infty}(\Omega)$. Then there is a $t_{0}\left(h_{1}\right) \in \mathbb{R}$ such that $\left(P_{t}\right)$ has at least one solution $u \in \widetilde{B}_{p, q}^{s}(\Omega)$ if $t<t_{0}$, but no solution if $t>t_{0}$.

(ii) Let $p<\infty$ and $h_{1} \in \widetilde{F}_{p, q}^{s-2}(\Omega) \cap L_{\infty}(\Omega)$. Then there is a $t_{0}\left(h_{1}\right) \in \mathbb{R}$ such that $\left(P_{t}\right)$ has at least one solution $u \in \tilde{F}_{p, q}^{s}(\Omega)$ if $t<t_{0}$, but no solution if $t>t_{0}$.

Proof. We consider (i). The proof of (ii) is the same.

Step 1. Assume one can solve $\left(P_{t_{1}}\right)$, and let $u$ be a solution. Then $u$ is a strict supersolution of $\left(P_{t}\right)$ for all $t<t_{1}$. By means of hypothesis (f2) and Franke and Runst [10, Theorem 3.4/1], we can find a strict subsolution $u_{-} \in \tilde{B}_{p, q}^{s}(\Omega)$ of $\left(P_{t}\right), u_{-}<u_{+}$. The following conditions ensure the existence of a subsolution $u_{-} \in \widetilde{B}_{p . q}^{s}(\Omega)$ of $\left(P_{t}\right)$ (see Franke and Runst [10, Subsection 3.4]):

There exists a real number $s_{-}$and a bounded $\tilde{C}^{\infty}$-function $h_{-}: \bar{\Omega} \times \mathbb{R} \rightarrow \mathbb{R}$ with

$$
\begin{aligned}
& h_{1}(x)+t \varphi_{1}(x)+f(v(x))-\lambda_{1} v(x) \geqq h_{-}(x, v(x)), \\
& \int_{\Omega} h_{-}(x, v(x)) \varphi_{1}(x) d x \geqq 0 \quad \text { if } \quad v \in \tilde{C}^{\infty}(\bar{\Omega}), \\
& v>s_{-} \varphi_{1} \text { in } \Omega
\end{aligned}
$$

By analogy with Drabek and Runst [7, Section 3] one can show: If $u_{+}$is a supersolution of $\left(P_{t}\right)$ and $u_{-}$is a subsolution of $\left(P_{t}\right), u_{-}<u_{+}$in $\Omega$, then there exists a function $u \in \widetilde{B}_{p, q}^{s}(\Omega)$ such that $u_{-} \leqq u \leqq u_{+}$in $\Omega$ and $u$ is a solution of $\left(P_{t}\right)$. Hence one can solve $\left(P_{t}\right)$ for all $t \leqq t_{1}$.

Step 2. We show that $t_{0}>-\infty$. It is enough to find for some $t \in \mathbb{R}$ a supersolution of $\left(P_{t}\right)$, since as in Step 1 we can then find a subsolution $u_{-}<u_{+}$. By our assumption we have $h_{1} \in \widetilde{B}_{p, q}^{s-2}(\Omega) \cap L_{\infty}(\Omega)$ and $f \in \tilde{C}^{p+1}$. Hence we can choose $t\left(h_{1}\right)<0$ so small such that $f(0)+h_{1}(x)+t \varphi_{1}(x)<0$ holds for $x \in \Omega$. Then $u_{+} \equiv 0$ is a supersolution of $\left(P_{t}\right)$.

Step 3. If we put $t_{0}=\sup \left\{t \in \mathbb{R},\left(P_{t}\right)\right.$ is solvable $\}$, then we get $t_{0}>-\infty$. To see that $t_{0}<\infty$, we apply hypothesis (f1). Let $u$ be a solution of $\left(P_{t}\right)$. Then we get

$$
\begin{aligned}
0 & =\int_{\Omega}\left(L u-\lambda_{1} u\right)(x) \varphi_{1}(x) d x \\
& =\int_{\Omega}\left(f(u)-\lambda_{1} u\right)(x) \varphi_{1}(x) d x+t
\end{aligned}
$$

which together with (f1) implies that $t_{0}<\infty$ holds. Our proof is finished. 
Remark 3.1. Notice that the above proof yields the folowing result: For each $h_{1} \in \widetilde{B}_{p, q}^{s}(\Omega) \cap L_{\infty}(\Omega)$ there exists a $t_{1}\left(h_{1}\right) \leqq t_{0}\left(h_{1}\right)$ such that $\left(P_{t}\right)$ has at least one negative solution for all $t \leqq t_{1}\left(h_{1}\right)$.

Remark 3.2. Theorem 3.1 is a generalization of the assertion in Kazdan and Warner [16, Corollary 3.11].

Now we consider the solvability of $\left(P_{t}\right)$ for $t=t_{0}\left(h_{1}\right)$. For it we need an additional condition on $f$. Then we can apply an idea similar to one used by Hess (see Lazer and McKenna [17, Section 3]).

Theorem 3.2. Let the assumption on $s, p, q$ and $\rho$ of Theorem 3.1 be satisfied and let $f \in \tilde{C}^{\rho+1}(\mathbb{R})$ satisfies the following conditions:

(f3) $f(x)-\lambda_{1} x \geqq c_{1}|x|-b$ for all $x \in \mathbb{R}$, where $c_{1}>0, b \geqq 0$ and $\lambda_{1}$ is, as usual, the first eigenvalue of $L$,

(f4) $f(x)$ is bounded on $[0, \infty)$.

Then there exists a solution $u_{0} \in \tilde{B}_{p, q}^{s}(\Omega)\left(u_{0} \in \tilde{F}_{p, q}^{s}(\Omega)\right)$ of $\left(P_{t}\right)$ when $t=t_{0}$.

Proof. We consider the case when $u_{0} \in \tilde{B}_{p, q}^{s}(\Omega)$ holds. The other part is almost the same.

Step 1. Let $\left\{t_{n}\right\}_{n=1}^{\infty} \subset \mathbb{R}, t_{n} \uparrow t_{0}$, and let $u_{n}$ be the corresponding solutions. Then $g_{n}(x)=h_{1}(x)+t_{n} \varphi_{1}(x)$ is bounded in $\widetilde{B}_{p, q}^{s-2}(\Omega) \cap L_{\infty}(\Omega)$. We prove that $u_{n}$ is bounded in $L_{\infty}(\Omega)$. Assume the contrary. Then there exists a sequence $\left\{g_{n}\right\}_{n=1}^{\infty}$ in $\widetilde{B}_{p, q}^{s-2}(\Omega) \cap L_{\infty}(\Omega)$ with $\left\|g_{n} \mid L_{\infty}\right\| \leqq M$ and a corresponding sequence $\left\{u_{n}\right\}_{n=1}^{\infty}$ in $\widetilde{B}_{p, q}^{s}(\Omega)$ satisfying $L u_{n}=$ $f\left(u_{n}\right)+g_{n}$ in $\Omega, u_{n}=0$ on $\partial \Omega$ and $\left\|u_{n} \mid L_{\infty}\right\| \rightarrow \infty$ as $n \rightarrow \infty$. By (f 3), we obtain, if $0<\lambda_{1}-\gamma<c_{1}$, the existence of a real number $c^{*}$ such that for all $n \geqq 1, L u_{n}-\gamma u_{n}=$ $g_{n}+\left(\lambda_{1}-\gamma\right) u_{n}+\left(f\left(u_{n}\right)-\lambda_{1} u_{n}\right) \geqq c^{*}$ on $\Omega$. By the properties of $L$ (see 2.2) there exists a function $v \in \widetilde{B}_{p, q, 0}^{s}(\Omega)$ satisfying $(L-\gamma) v=c^{*}$. Since $\gamma<\lambda_{1}$, Lemma 2.1 implies $u_{n}(x) \geqq$ $\min _{x \in \Omega} v(x)$ for $x \in \Omega$ and all $n$.

If we define $w_{n}=u_{n} /\left\|u_{n} \mid L_{\infty}\right\|$, from (f4) it follows for some $K>0,\left\|L w_{n} \mid L_{\infty}\right\|<K$ for all $n \geqq 1$. By the mapping properties of $L$ and compactness results, see 2.1 , it follows that we may assume $w_{n} \rightarrow w$ in $L_{\infty}(\Omega)$. Then $\left\|w \mid L_{\infty}\right\|=1$ and since

$$
w_{n}(x) \geqq \frac{\min v(x)}{\left\|u_{n} \mid L_{\infty}\right\|},
$$

we obtain $w(x) \geqq 0$ in $\Omega$.

On the other hand, by (f3) we have

$$
0=\int_{\Omega}\left(L w_{n}-\lambda_{1} w_{n}\right)(x) \varphi_{1}(x) d x
$$




$$
\begin{aligned}
& =\frac{1}{\left\|u_{n} \mid L_{\infty}\right\|}\left(\int_{\Omega}\left[f\left(u_{n}\right)-\lambda_{1} u_{n}\right](x) \varphi_{1}(x) d x+\int_{\Omega} g_{n}(x) \varphi_{1}(x) d x\right) \\
& \geqq \frac{1}{\left\|u_{n} \mid L_{\infty}\right\|}\left(\int_{\Omega} c_{1}\left|u_{n}(x)\right| \varphi_{1}(x) d x+\int_{\Omega}\left(g_{n}(x)-b\right) \varphi_{1}(x) s x\right) .
\end{aligned}
$$

Hence

$$
\begin{aligned}
\int_{\Omega} w_{n}(x) \varphi_{1}(x) d x & =\frac{1}{\| u_{n}\left|L_{\infty}\right| \mid} \int_{\Omega} u_{n}(x) \varphi_{1}(x) d x \\
& \leqq \frac{1}{\left\|u_{n} \mid L_{\infty}\right\|} \int_{\Omega}\left|u_{n}(x)\right| \varphi_{1}(x) d x \\
& \leqq \frac{1}{c_{1}\left\|u_{n} \mid L_{\infty}\right\|} \int_{\Omega}\left(b-g_{n}(x)\right) \varphi_{1}(x) d x
\end{aligned}
$$

Now $n \rightarrow \infty$, implies a contradiction to $w(x) \geqq 0$ in $\Omega,\left\|w \mid L_{\infty}\right\|=1$.

Step 2. We show that $\left\{u_{n}\right\}_{n=1}^{\infty}$ is also bounded in $\widetilde{B}_{p, q}^{s}(\Omega)$. Let $0<\varepsilon<2$ be small enough such that $s-\varepsilon>(n / p)$. Step 1 and Lemma 2.2 yield $\left\|f\left(u_{n}\right) \mid B_{p, q}^{s-2}\right\| \leqq$ $c_{2}\left(1+\left\|u_{n} \mid B_{p, q}^{s-\varepsilon}\right\|\right)$. Because of the imbedding $L_{\infty}(\Omega) \subset B_{p, 2}^{0}(\Omega)$, see 2.1 , and the inequality $\left\|g\left|B_{p, q}^{\theta_{s 0}+\left(1-f_{)} s_{1}\right.}\left\|\leqq c_{3}\right\| g\right| B_{p, 2}^{s_{0}}\right\|\left\|^{\theta}\right\| g\left|B_{p, q}^{s_{1}} \|\right|^{1-\theta}, 0<\theta<1$, we get from $L u_{n}=f\left(u_{n}\right)+g_{n}$

$$
\begin{aligned}
\left\|u_{n} \mid B_{p, q}^{s}\right\| & \leqq c_{4}\left(\left\|g_{n}\left|B_{p, q}^{s-2}\|+\| f\left(u_{n}\right)\right| B_{p, q}^{s-2}\right\|\right) \\
& \leqq c_{5}\left(1+\left\|u_{n} \mid B_{p, q}^{s}\right\|^{\theta}\right) .
\end{aligned}
$$

Now we conclude $\left\|u_{n} \mid B_{p, q}^{s}\right\| \leqq M_{1}$. This proves the boundedness of $\left\{u_{n}\right\}_{n=1}^{\infty}$ in $\widetilde{B}_{p, q}^{s}(\Omega)$.

Step 3. We have proved that the solutions $u_{n}$ of $u_{n}=L^{-1}\left[f\left(u_{n}\right)+h_{1}(x)+t_{n} \varphi_{1}(x)\right]$, $t_{n} \uparrow t_{0}$, are bounded in $\widetilde{B}_{p, q}^{s}(\Omega)$. Applying compactness arguments, we see $u_{n} \rightarrow u$ in $\tilde{B}_{p, q, 0}^{s}(\Omega)$ and $u$ is a solution of $\left(P_{t}\right)$ when $t=t_{0}$. Our proof is finished.

Remark 3.3. Theorem 3.2 generalizes a result obtained by Amann and Hess [1] (see also Dancer [4] and Lazer and McKenna [17]).

\subsection{Multiplicity results}

In this subsection, we prove results concerning the number of solutions of the problem $\left(\boldsymbol{P}_{t}\right)$ within the framework of Besov and Triebel-Lizorkin spaces.

Lemma 3.1. Let $0<p, q \leqq \infty, 2>s>(n / p), \rho>\max (1, s)$ and $f \in \tilde{C}^{\rho+1}(\mathbb{R})$. If $t_{1}<t<t_{2}$ 
and if for $i=1,2, u_{i}(x)$ is a solution of $\left(P_{t}\right)$ when $t=t_{i}$ such that $u_{1}(x) \leqq u_{2}(x)$ then there exists a number $r$ such that

$$
d_{\mathrm{LS}}\left(u-L^{-1}\left(f(u)+h_{1}+t \varphi_{1}\right), \operatorname{Int} K, 0\right)=1
$$

where

$$
K=\left\{u \in \widetilde{B}_{p, q, 0}^{s}(\Omega), u_{1} \leqq u \leqq u_{2} \text { in } \Omega,\left\|u \mid B_{p, q}^{s}\right\| \leqq r\right\}
$$

Remark 3.4. A corresponding result holds also in Triebel-Lizorkin spaces.

Proof of Lemma 3.1. We use an argument of Fucik [11, Theorem 34.7] (see also Lazer and McKenna [17]). By our assumption we have

$$
L u_{1}<f\left(u_{1}\right)+h_{1}+t \varphi_{1} \text { in } \Omega, u_{1} \mid \partial \Omega=0,
$$

and

$$
L u_{2}>f\left(u_{2}\right)+h_{1}+t \varphi_{1} \text { in } \Omega, u_{2} \mid \partial \Omega=0 .
$$

By Theorem 3.1, Step 1 we obtain that there is a solution $u \in \widetilde{B}_{p, q, 0}^{s}(\Omega)$ satisfying $\left(P_{t}\right)$ and $u_{1} \leqq u \leqq u_{2}$. By analogy with Drabek and Runst [7, Section 3], we choose a number $\omega>0$ such that $\omega+f^{\prime}(\xi)>0$ for $\xi \in\left[\min _{x \in \bar{\Omega}} u_{1}(x), \max _{x \in \bar{\Omega}} u_{2}(x)\right]$. Notice that $\tilde{B}_{p, q}^{s}(\Omega) \subset$ $\tilde{C}(\bar{\Omega})$ if $s>(n / p)$. Then we get

$$
(L+\omega)\left(u_{2}-u\right)=f\left(u_{2}\right)-f(u)+\omega\left(u_{2}-u\right)+\left(t_{2}-t_{1}\right) \varphi_{1}
$$

and

$$
(L+\omega)\left(u_{2}-u-\left(\frac{t_{2}-t}{\omega+\lambda_{1}}\right) \varphi_{1}\right)=f\left(u_{2}-f(u)+\omega\left(u_{2}-u_{1}\right)>0\right.
$$

Since $u\left|\partial \Omega=u_{2}\right| \partial \Omega=\varphi_{1} \mid \partial \Omega=0$, Lemma 2.1 implies $u_{2}(x)>u(x)$ for $x \in \Omega$. Analogously, we can prove $u_{1}(x)<u(x)$ for $x \in \Omega$. Now we define $f: \Omega \times \mathbb{R} \rightarrow \mathbb{R}$ by

$$
f(x, u)= \begin{cases}f\left(u_{2}(x)\right)+h_{1}(x)+t \varphi_{1}(x) & \text { if } u \geqq u_{2}(x) \\ f(u)+h_{1}(x)+t \varphi_{1}(x) & \text { if } \quad u_{1}(x) \leqq u \leqq u_{2}(x) \\ f\left(u_{1}(x)\right)+h_{1}(x)+t \varphi_{1}(x) & \text { if } u \leqq u_{1}(x) .\end{cases}
$$

Notice that by our assumptions $L_{p_{1}}(\Omega) \subset B_{p, q}^{s-2+\varepsilon}(\Omega)$ for some $p_{1}<1$ and $\varepsilon>0$ sufficiently small. By definition of $\widetilde{f}$ it holds that $L^{-1} \tilde{f}$ is completely continuous in $\tilde{B}_{p, q, 0}^{s}(\Omega)$ and $\left\|L^{-1} f(x, u) \mid B_{p, q}^{s}\right\|<R$ for $u \in \widetilde{B}_{p, q, 0}^{s}(\Omega)$ if $R$ is chosen sufficiently large. For it we apply 
the same arguments as in Step 2 of Theorem 3.2. Furthermore, Lemma 2.1 shows that $u=L^{-1} \widetilde{f}(x, u)$ implies $u \in \operatorname{Int} K$. Now standard arguments prove

$$
\begin{aligned}
1 & =d_{\mathrm{LS}}\left(u, B_{R}(0), 0\right)=d_{\mathrm{LS}}\left(u-L^{-1} f(x, u), B_{R}(0), 0\right) \\
& =d_{\mathrm{LS}}\left(u-L^{-1}\left(f(u)+h_{1}+t \varphi_{1}\right), \operatorname{Int} K, 0\right),
\end{aligned}
$$

where Int $K \subset B_{R}(0)=\left\{u \in \widetilde{B}_{p, q, 0}^{s}(\Omega),\left\|u \mid B_{p, q}^{s}\right\|<R\right\}$.

Lemma 3.2. Let all the hypotheses of Theorem 3.2 be satisfied and $t$ be a real number.

(i) Let $B_{R}(0)=\left\{u \in B_{p, q, 0}^{s}(\Omega),\left\|u \mid B_{p, q}^{s}\right\|<R\right\}$. Then there exists $R_{0}=R_{0}(t)>0$ such that

$$
d_{\mathrm{LS}}\left(u-L^{-1}\left(f(u)+h_{1}+t \varphi_{1}\right), B_{R_{1}}(0), 0\right)=0
$$

for $R_{1}>R_{0}$.

(ii) Let $p<\infty$ then a corresponding result is also true for $\tilde{F}_{p, q}^{s}$.

Proof. We use an argument of Dancer [4] and Theorem 3.2. Let $t_{1}>t_{0}\left(h_{1}\right)$ and let $R_{0}$ be sufficiently large such that

$$
\begin{array}{ll}
L u=f(u)+h_{1}(x)+\left[t+s\left(t_{1}-t\right)\right] \varphi_{1}(x) & \text { in } \Omega \\
u=0 & \text { on } \partial \Omega, 0 \leqq s \leqq 1,
\end{array}
$$

imply $u \in B_{R_{0}}(0)$. By Theorem 3.1 we know that there is no solution for $s=1$. Hence, we obtain by the a priori bound and the homotopy invariance

$$
\begin{aligned}
0 & =d_{\mathrm{LS}}\left(u-L^{-1}\left(f(u)+h_{1}+t_{1} \varphi_{1}\right), B_{R_{1}}(0), 0\right) \\
& =d_{\mathrm{LS}}\left(u-L^{-1}\left(f(u)+h_{1}+t \varphi_{1}\right), B_{R_{1}}(0), 0\right)
\end{aligned}
$$

for $R_{1}>R_{0}(t)$

In the following, we describe the existence of at least three solutions of $\left(P_{t}\right)$.

Theorem 3.3. Let $0<p, q \leqq \infty, 2>s>(n / p)$ and $f \in \tilde{C}^{\rho+1}(\mathbb{R}), \rho>\max (1, s)$, satisfying (f3) and $\lim _{x \rightarrow+\infty} f^{\prime}(x)=\alpha$, where $\lambda_{2}<\alpha<\lambda_{3}$, and $\lambda_{2}$ has multiplicity one.

(i) Let $h_{1} \in \tilde{B}_{p, q}^{s-2}(\Omega) \cap L_{\infty}(\Omega)$. Then there exists a $t_{1}\left(h_{1}\right) \in \mathbb{R}$ such that if $t>t_{1},\left(P_{t}\right)$ has at least three solutions.

(ii) Let $p<\infty$ and $h_{1} \in \tilde{F}_{p, q}^{s-2}(\Omega) \cap L_{\infty}(\Omega)$. Then there exists a $t_{1}\left(h_{1}\right) \in \mathbb{R}$ such that $\left(P_{t}\right)$ has at least three solutions if $t<t_{1}$. 
Proof. We give an outline of the proof of (i). The other part is almost the same. We apply a method used in Lazer and McKenna [17, Lemma 3.7].

Step 1. Let $z(x)=\varphi_{1}(x) /\left(\lambda_{1}-\alpha\right)$. Since $z<0$ in $\Omega, \partial z / \partial v>c>0$ on $\partial \Omega$, there exists $\delta_{1}>0$ such that if $v \in \tilde{B}_{p, q, 0}^{s}(\Omega)$ then $\left\|v-z \mid B_{p, q}^{s}\right\|<\delta_{1}$ implies $v<0$ in $\Omega$. Here we used $\tilde{B}_{p, q}^{s}(\Omega) \subset \tilde{C}(\bar{\Omega})$ if $s>(n / p)$. Let $\delta>0$ be given. Then we choose some $x_{0} \in \Omega$ and put $r=\min \left(\delta, \delta_{1},\left(-z\left(x_{0}\right)\right) / 2\right.$. The mapping $u \rightarrow L^{-1}\left(\varphi_{1}+\alpha u\right)$ is completely continuous on $\widetilde{B}_{p, q, 0}^{s}(\Omega)$. Since $z=L^{-1}\left(\varphi_{1}+\alpha z\right)$ holds, we obtain that there exists $\eta>0$ such that $\left\|u-z \mid B_{p, q}^{s}\right\|=r$ implies $\left\|u-L^{-1}\left(\varphi_{1}+\alpha u\right) \mid B_{p, q}^{s}\right\| \geqq \eta$. Applying Corollary 2.1 and Theorem 2.1 , we get

$$
d_{\mathrm{LS}}\left(u-L^{-1}\left(\varphi_{1}+\alpha u\right), V_{r}, 0\right)=1,
$$

where $V_{r}=\left\{u \in \widetilde{B}_{p, q, 0}^{s}(\Omega),\left\|u-z \mid B_{p, q}^{s}\right\|<r\right\}$. (Notice that $\alpha L^{-1}$ has precisely two eigenvalues larger than 1.)

Step 2. Now we consider the boundary value problem

$$
\begin{array}{ll}
L u=\frac{f(t u)-\alpha t u}{t}+\varphi_{1}+\frac{h_{1}}{t}+\alpha u & \text { in } \Omega \\
u=0 & \text { on } \partial \Omega
\end{array}
$$

Notice that $u$ is a solution of $\left(P_{t}\right)$ if and only if $t u$ is a solution of (3.4). We get for $t<0$

$$
\begin{aligned}
& \| L^{-1}\left[\varphi_{1}+\alpha u+\frac{1}{t}\left(h_{1}+(f(t u)-\alpha t u)\right]-L^{-1}\left[\varphi_{1}+\alpha u\right] \mid B_{p, q}^{s} \|\right. \\
& \quad=\left\|L^{-1}\left[\frac{1}{t}\left(h_{1}+(f(t u)-\alpha t u)\right)\right] \mid B_{p, q}^{s}\right\| \\
& \quad \leqq c\left\|\frac{1}{t}\left(h_{1}+(f(t u)-\alpha t u)\right) \mid L_{p_{1}}\right\|
\end{aligned}
$$

for some $p_{1}>1$. Here we used the mapping properties of $L$ and imbedding results (see 2.1/2.2).

From $\lim _{s \rightarrow \infty} f^{\prime}(s)=\alpha$ we infer that for $t \leqq T<0\left\|u-z \mid B_{p, q}^{s}\right\|<r$ (which implies $u<0$ in $\Omega)$ the following estimate holds:

$$
c\left\|\frac{1}{t}\left(h_{1}+(f(t u)-\alpha t u)\right) \mid L_{p_{1}}\right\|<\eta .
$$


Hence by Step 1, from (3.3), (3.6) it follows that

$$
\begin{aligned}
1 & =d_{\mathrm{LS}}\left(u-L^{-1}\left[\varphi_{1}+\alpha u\right], V_{r}, 0\right) \\
& =d_{\mathrm{LS}}\left(u-L^{-1}\left[\varphi_{1}+\alpha u+\frac{1}{t}\left(h_{1}+f(t u)-\alpha t u\right)\right], V_{r}, 0\right)
\end{aligned}
$$

(homotopy invariance). This implies the existence of a solution $v \in \widetilde{B}_{p, q, 0}^{s}(\Omega)$ of $(3.10)$ satisfying $\left\|v-z \mid B_{p, q}^{s}\right\|<r$. Then $t v$ is a solution of $\left(P_{t}\right)$. Hence we have shown that for given $\delta>0$ there exists $T(\delta)$ so that if $t<T(\delta),\left(P_{t}\right)$ has a solution $w$ such that $\left\|z-(w / t) \mid B_{p, q}^{s}\right\|<\delta$. Similarly, one can prove

$$
\begin{aligned}
1 & =d_{\mathrm{LS}}\left(u-L^{-1}\left[t \varphi_{1}-\alpha u\right], B(t), 0\right) \\
& =d_{\mathrm{LS}}\left(u-L^{-1}\left[t \varphi_{1}+h_{1}+f(u)\right], B(t), 0\right),
\end{aligned}
$$

where $B(t)=\left\{u \in \widetilde{B}_{p, q, 0}^{s}(\Omega),\left\|u-t z\left|B_{p, q}^{s} \|<\right| t \mid r\right\}\right.$. Notice that $u \in B(t)$ and $r \geqq-\left(z\left(x_{0}\right) / 2\right)$, where $x_{0} \in \Omega$, implies $u\left(x_{0}\right)>c\left(t z\left(x_{0}\right) / 2\right)$. Since $z\left(x_{0}\right)<0$ and since $u \in K(t)$ implies $u\left(x_{0}\right) \leqq u_{2}\left(x_{0}\right)$, where $K$ and $u_{2}$ as in Lemma 3.1 we get

$$
\overline{B(t)} \cap K(t)=\varnothing
$$

for $t<T(\delta)$ and some fixed $r \in(0, \delta)$.

Step 3. For given $\delta>0$ we choose $t<T(\delta)$ such that (3.9) holds. Now we can find by Lemma $3.2 B_{R}(0) \supset(\overline{B(t)} \cup K(t))$ such that $(3.3)$ is satisfied. By the properties of the Leray-Schauder degree (Lemma 2.5(c)) we deduce that

$$
d_{\mathrm{LS}}\left(u-L^{-1}\left[t \varphi_{1}+h_{1}+f(u)\right], B_{R}(0) \backslash(\overline{B(t)} \cup K(t)), 0\right) \neq 0 .
$$

Hence in each of the three disjoint sets $B(t)$, Int $K(t)$ and $B_{R}(0) \backslash(\overline{B(t)} \cup K(t))$ there exists at least one solution of $\left(P_{t}\right)$. This completes the proof.

Remark 3.5. The conclusion of Theorem 3.3 holds also if $\lambda_{2 m}<\alpha<\lambda_{2 m+1}$. In the proof of (3.7), (3.8) we use the homotopy invariance of the Leray-Schauder degree. Therefore it was essential that (3.5) and (3.6) hold. In general,

$$
\left\|\frac{1}{t}\left(h_{1}+(f(t u)-\alpha t u)\right) \mid B_{p, q}^{s-2}\right\|<\eta
$$

does not hold for $t<T,\left\|u-z \mid B_{p, q}^{s}\right\| \leqq r$ and $u \leqq 0$ in $\bar{\Omega}$ if $s-2>(n / p)\left(\tilde{B}_{p, q}^{s-2}(\Omega)\right) \subset \tilde{C}(\bar{\Omega})$. A simple counter-example is $f(x) \sim c|x|^{m}$ near the origin and $\lim _{s \rightarrow \infty} f^{\prime}(s)=\alpha$ (see Runst [22, Subsection 5.4]).

Remark 3.6. If $\lambda_{1}<f^{\prime}(+\infty)<\infty$ holds then we get by Lemma 3.1 and Lemma 3.2 
that $\left(P_{t}\right)$ admits at least two solutions if $t<t_{0}\left(h_{1}\right)$. It is a generalization of a result obtained by Amann and Hess [1] (see also Dancer [4]).

\section{REFERENCES}

1. H. Amman and P. Hess, A multiplicity result for a class of elliptic boundary value problems, Proc. Roy. Soc. Edinburgh Sect. A (1979), 145-151.

2. A. Ambrosetti and G. Prodl, On the inversion of some differentiable mappings with singularities between Banach spaces, Ann. Mat. Pura Appl. 93 (1972), 231-246.

3. M. S. Berger and E. Podolak, On the solutions of a nonlinear Dirichlet problem, Indiana Univ. Math. J. 24 (1975), 837-849.

4. E. N. Dancer, On the ranges of certain weakly nonlinear elliptic partial differential equations, J. Math. Pure Appl. 57 (1978), 351-366.

5. K. Deimuing, Nonlinear Functional Analysis (Springer-Verlag, Berlin/Heidelberg/New York/ Tokyo, 1985).

6. C. L. Dolph, Nonlinear integral equations of the Hammerstein type, Trans. Amer. Math. Soc. 60 (1949), 289-307.

7. P. Drabek and T. Runst, On the existence of solutions of a semilinear elliptic boundary value problem with superlinear nonlinearities, Z. Anal. Anwendungen, to appear.

8. J. Franke, Regular elliptic boundary value problems in Besov and Triebel-Lizorkin spaces. The case $0<p \leqq \infty, 0<q \leqq \infty$, preprint.

9. J. Franke and T. RUNST, On the admissibility of function spaces of type $B_{p, q}^{s}$ and $F_{p, q}^{s}$, and boundary value problems for non-linear partial differential equations, Anal. Math. 13 (1987), 3-27.

10. J. Franke and T. Runst, Non-linear perturbations of linear non-invertible boundary value problems in function spaces of type $B_{p, q}^{s}$ and $F_{p, q}^{s}$, Czechoslovak Math. J. 38 (113) (1988), 623-641.

11. S. Fucik, Solvability of Nonlinear Equations and Boundary Value Problems (Soc. Czechoslovak Math. Phys., Prague, 1980).

12. M. Geisler and T. Runst, On a superlinear Ambrosetti-Prodi problem in Besov and Triebel-Lizorkin spaces, to appear.

13. P. Hess and B. Ruf, On a superlinear elliptic boundary value problem, Math. Z. 164 (1978), 9-14.

14. V. KLEE, Leray-Schauder theory without local convexity, Math. Ann. 141 (1960), 281-285.

15. G. Котне, Topologische lineare Räume, I (Springer-Verlag, Berlin/Heidelberg/New York, 1960).

16. J. L. Kazdan and F. W. WARnER, Remarks on some quasi-linear elliptic equations, Comm. Pure Appl. Math. 28 (1975), 567-597.

17. A. C. Lazer and P. J. McKenna, On the number of solutions of a nonlinear Dirichlet problem, J. Math. Anal. Appl. 84 (1981), 282-294.

18. A. C. Lazer and P. J. McKenna, Multiplicity of solutions of nonlinear boundary value problems with nonlinearities crossing several higher eigenvalues, J. Reine Angew. Math. 368 (1986), 184-200. 
19. A. Manes and A. M. Micheletti, Un' estensione della teoria variazionale classica degli autovalori per operatori ellittici del secondo ordine, Boll. Un. Mat. Ital. 7 (1973), 285-301.

20. T. RIEDRICH, Vorlesungen über nichtlineare Operatorengleichungen (Teubner-Texte Math. Teubner, Leipzig, 1976).

21. B. RuF, On nonlinear elliptic boundary value problems with jumping nonlinearities, Ann. Math. Pura Appl. 128 (1980), 131-151.

22. T. RUNST, Mapping properties of non-linear operators in spaces of Triebel-Lizorkin and Besov type, Analysis Math. 12 (1986), 313-346.

23. S. Solimini, Some remarks on the number of solutions of some nonlinear elliptic problems, Ann. Inst. H. Poincaré 2 (1985), 143-156.

24. H. Triebel, Theory of Function Spaces (Geest and Porting, Leipzig, 1983; Birkhäuser, Basel, 1983).

25. H. TRIEBEL, Mapping properties of non-linear operators generated by $\Phi(u)=\left.\left.\right|_{u}\right|^{\rho}$ and by holomorphic $\Phi(u)$ in function spaces of Besov-Hardy-Sobolev type. Boundary value problems for elliptic differential equations of type $\Delta u=f(x)+\Phi(u)$, Math. Nachr. 117 (1984), 193-213.

26. J. H. Wiluamson, Compact linear operators in linear topological spaces. J. London Math. Soc. 29 (1954), 149-156.

27. E. ZEIDLER, Vorlesungen über nichtlinear Funktionalanalysis I-Fixpunktsätze (Teubner-Texte Math., Teubner, Leipzig, 1976).

Department of Mathematics

UNIVERSITY OF HELSINKI

Hallituskatu 15

SF-00100 HelsinkI 10

FinLand
Section Mathematik

UNIVERSITÄT JENA

UNIVERSITÄTSCHOCHHAUS

DDR-6900 JENA

Germany 